\title{
No PTSD-related differences in diurnal cortisol profiles of genocide survivors
}

\author{
Cindy Eckart ${ }^{a, *}$, Harald Engler ${ }^{b, c}$, Carsten Riether ${ }^{b}$, Stephan Kolassa ${ }^{d}$, \\ Thomas Elbert ${ }^{a}$, Iris-Tatjana Kolassa ${ }^{a, e, * *}$
}

\author{
${ }^{a}$ Clinical Psychology \& Neuropsychology, Department of Psychology, University of Konstanz, Germany \\ ${ }^{\mathrm{b}}$ Psychology \& Behavioral Immunobiology, Institute for Behavioral Sciences, ETH Zurich, Switzerland \\ "Institute of Medical Psychology \& Behavioral Immunobiology, University Hospital of Essen, \\ University of Duisburg-Essen, Germany \\ ${ }^{d}$ Research \& Innovation, SAF Simulation, Analysis \& Forecasting AG, Tägerwilen, Switzerland \\ 'Zukunftskolleg, University of Konstanz, Box X 916, 78457 Konstanz, Germany
}

\author{
KEYWORDS \\ Cortisol; \\ HPA axis; \\ Posttraumatic stress \\ disorder; \\ PTSD; \\ Stress; \\ Building-block effect
}

\begin{abstract}
Summary Posttraumatic stress disorder (PTSD) has been associated with reduced cortisol levels. Opposing results have been interpreted as resulting from methodological differences between studies. We investigated the diurnal profile of salivary cortisol in a population of highly traumatized adult males from Rwanda with and without PTSD, who spent the whole day of examination together under a maximally standardized schedule. Besides the detection of PTSDrelated alterations in cortisol release we aimed at determining physiologically relevant effects of cumulative trauma exposure on HPA functioning in interaction with or independent of diagnosis.

There were no differences in the diurnal pattern of cortisol release between subjects with and without PTSD. We observed an increasing prevalence of PTSD with increasing number of different traumatic event types experienced, replicating earlier results on a "building-block effect" of multiple traumatization. However, size of cumulative exposure was not related to any of the cortisol measures.

The results suggest that besides methodological constraints also confounding factors not previously controlled for, e.g., sex differences or current life stress, might contribute to the diverging results of lowered, unchanged or enhanced cortisol secretion in PTSD. Future research should therefore closely monitor these possible confounds to optimize models for cortisol in research on stress-dependent illnesses.
\end{abstract}

\footnotetext{
* Corresponding author at: University of Konstanz, Universitätsstr. 10, 78457 Konstanz, Germany. Tel.: +49 7531883086 ; fax: +497531 884601.

** Corresponding author at: University of Konstanz, Universitätsstr. 10, 78457 Konstanz, Germany. Tel.: +49 7531884003 ; fax: +49 7531884601 . E-mail addresses: cindy.eckart@uni-konstanz.de (C. Eckart), iris.kolassa@uni-konstanz.de (I.-T. Kolassa).
} 


\section{Introduction}

The hypothalamus-pituitary-adrenal (HPA) axis is one of the key systems mediating the physiological reactions to acute and chronic stress (McEwen, 2000; Smith and Vale, 2006). Increased cortisol concentrations have been shown subsequent to multiple psychological and physical stressors (for a review see Kirschbaum and Hellhammer, 1994). In the short run an enhanced secretion of cortisol promotes adaptation to the challenges of the stressor through a process known as allostasis. In the long run, however, repeated stress might cumulate to a dysregulation of endocrinological mechanisms referred to as allostatic (over)load (McEwen, 2005). In line with this theoretical framework, chronically elevated cortisol levels were found in populations reporting continuously high life stress (Luecken et al., 1997; Powell et al., 2002) and in men of low socioeconomic status (Steptoe et al., 2003; Cohen et al., 2006). Furthermore, it has been suggested that sustained high life stress may lead to an enhanced cortisol variation (Kaspers and Scholz, 2004), which might partly be due to inter-individual differences in the reactivity to and recovery from stress (Kirschbaum et al., 1995; Roy et al., 2001).

Posttraumatic stress disorder (PTSD) is a psychiatric condition that may emerge in the aftermath of a potentially lifethreatening experience. Since traumatic experiences imply an extreme stress for the organism, it has been suggested that persistent alterations in HPA axis functions might also be involved in the pathophysiology of PTSD. A substantial amount of research has focused on the diurnal profile of cortisol secretion in PTSD. Most of these studies reported lowered cortisol levels in subjects with PTSD: in a chronobiological analysis, a diminished cortisol secretion in PTSD patients was reported especially during the late evening and early morning hours (Yehuda et al., 1996). In an attempt to replicate these findings in a geriatric sample, Yehuda et al. (2005) found a slightly different pattern: elderly subjects with PTSD showed lowered cortisol levels at the time of awakening and at 08:00 h but increased salivary cortisol at $20: 00 \mathrm{~h}$, resulting in a flattened diurnal pattern of cortisol release (Yehuda et al., 2005). This pattern, in conjunction with an overall reduction of cortisol levels, has also been observed in Croatian (Lauc et al., 2004) and Bosnian (Rohleder et al., 2004) war refugees. Taken together, these data provide evidence for a diminished cortisol secretion in PTSD.

However, several studies have found opposing results as well. The diurnal cortisol profiles of women formerly subjected to childhood sexual abuse (Altemus et al., 2003) and of subjects from a low-income community (Young and Breslau, 2004; Young et al., 2004) showed no PTSD-related differences at all. Elevated cortisol levels on the other hand were found in women abused by their intimate partner (Inslicht et al., 2006). So far these discrepancies have mainly been attributed to methodological differences between studies. Co-morbid psychiatric illnesses, substance abuse, current medication, the time interval since traumatization and deficiencies in the standardization of the daily schedule of the subjects might interfere with PTSD-related endocrinological alterations (Rasmusson et al., 2003).

Furthermore the extent of traumatization might affect the cortisol release as well. A strong correlation has been documented between the number of different traumatic events reported by the subject and the diagnosis as well as symptom severity of PTSD (building-block effect; Neuner et al., 2004, 2006; Dohrenwend et al., 2006; Kolassa and Elbert, 2007). This finding might be interpreted in line with the assumption that repeated stress might enhance the allostatic load of an individual, resulting in increasing biological (e.g. cardiovascular and endocrinological dysregulation) and behavioral (e.g. antisocial responses, risk taking behaviors) consequences for the organism (McEwen, 2000; McEwen, 2005). Referring to this theoretical framework the cumulative exposure to traumatic events in interaction with or independent of PTSD might not only be reflected in stronger PTSD symptoms but might also alter the secretion of cortisol. This has also been supposed by Friedman et al. (2007) who investigated the 24-h urinary cortisol profile of women suffering from PTSD due to childhood sexual abuse. Women who were recurrently abused during their adulthood showed elevated cortisol levels compared to women without a history of repeated traumatization (Friedman et al., 2007).

In this study, we investigated the diurnal profile of cortisol release in a population of adult African refugees who had fled during the time of the Rwandan genocide (1994) to the Nakivale refugee camp in south-western Uganda. We exclusively concentrated on male subjects, as changes of basal saliva cortisol during the menstrual cycle cannot be ruled out (Kirschbaum et al., 1999). Our population is particularly homogenous, differing from those examined in previous studies in that all participants had (1) repeatedly experienced very similar traumatic events during the Rwandan genocide, (2) shared similar life circumstances before and during execution of the study, (3) received no psychiatric medication, (4) lived under natural circadian day-night rhythms (due to a lack of electricity in the camp), (5) showed almost no use of psychoactive substances (due to limited availability), (6) spent the whole day during the examination together in a maximally standardized daily schedule including food intake and rest, and (7) gave their saliva samples at exactly the same time. This design allowed us an exact monitoring of methodological factors previously proposed as reasons for the inconsistent findings in the endocrinological research on PTSD. As our non-PTSD control group was traumatized as well, we should furthermore be able to detect physiologically relevant effects of cumulative trauma exposure on HPA functioning (i.e. a building-block effect of trauma load on cortisol levels) in interaction with or independent of PTSD diagnosis.

\section{Methods}

\subsection{Setting}

Sixty male refugees participated in the study. All subjects had experienced traumatic events in conjunction with the Rwandan genocide in 1994 and subsequent persecution by Rwandan officials. Diagnostic procedures took place in the preparation phase of the study. Participants remained one entire day together at a designated place (in groups on three consecutive days) for cortisol specimen collection. Prior to the beginning of the study, the purpose of the investigation was explained in detail and informed consent was acquired. The study was conducted in accordance with the Declaration 
of Helsinki and approved by the Ethics Committee of the University of Konstanz, Germany, as well as by the Ethics Committee of the Mbarara University of Science and Technology, Uganda. Participants received an adequate compensation, corresponding to a day's salary.

\subsection{Diagnostic interviews}

Participants were interviewed and diagnosed in the preparation phase of the study. Interviews were structured and administered in the native language of Kinyarwanda. If necessary, trained interpreters were used. Local interviewers and interpreters that had been trained in concepts and diagnosis of mental illness with focus on PTSD in earlier studies (the selection and training procedure was described in detail Onyut et al., 2004; Neuner et al., 2008) were chosen from the refugee community and retrained and evaluated in conjunction with an earlier epidemiological study ( $\mathrm{Ertl}, 2005)$. Two psychologists, specially trained and experienced in the diagnosis of PTSD in survivors of organized violence, organized and supervised the data collection and the study on-site.

Sociodemographic information was obtained and subjects were further questioned about the occurrence of a subset of illnesses frequently displayed in East Africa. Additional information about their nutritional status, smoking habits, and average consumption of alcohol and other drugs was also obtained.

\subsubsection{Nakivale Event Checklist}

The extent of traumatization was evaluated with the Nakivale Event Checklist (Neuner et al., 2004), a checklist especially developed for the assessment of traumatization in survivors of the Rwandese genocide. This scale is based on an unweighted sum of 31 war-related and nonwar-related traumatic event types (e.g. witnessing the murder of a relative, imprisonment, torture, being harassed by officials, or experiencing an accident). A validation of the Nakivale Event Checklist in conjunction with an earlier epidemiological study (Ertl, 2005) revealed a satisfactory internal consistency (Cronbach's Alpha $=0.88$ ) in 89 Rwandese refugees.

\subsubsection{Posttraumatic Diagnostic Scale}

Current and lifetime PTSD symptoms were assessed using the Posttraumatic Diagnostic Scale (PDS; Foa et al., 1997), a measure that has already been used and validated in comparable African populations (Neuner et al., 2004; Odenwald et al., 2007). The PDS is a 17-item questionnaire (scoring from 0 to 51) that allows a quantification of the three clusters of PTSD symptoms (intrusions, avoidance, hyperarousal). Foa and colleagues reported a Cronbach's Alpha of 0.92 in a sample of 248 traumatized subjects (Foa et al., 1997). In an African population of 135 Somali ex-combatants the instrument achieved a Cronbach's Alpha of 0.86 (Odenwald et al., 2007).

\subsubsection{Hopkins Symptom Checklist-25}

Subjects were asked about symptoms of anxiety and depression with the Hopkins Symptom Checklist-25 (HSCL; Derogatis et al., 1974). The 25 items of the HSCL represent 10 core symptoms of anxiety and 15 core symptoms of depression which are rated according to their severity in the past week.
Anxiety and depression scores are then calculated as the sum of items divided by the number of items answered. In a Tanzanian population of 787 antenatal women (Lee et al., 2008 ), the HSCL yielded Cronbach's Alphas of 0.90 for the total score, 0.88 for the depression subscale and 0.76 for the anxiety subscale.

\subsubsection{Mini-International Neuropsychiatric Interview}

Diagnosis of Major Depression and suicidal ideation according to DSM-IV (American Psychiatric Association, 1994) took place using the corresponding sections of the Mini-International Neuropsychiatric Interview (M.I.N.I.; Sheehan et al., 1998).

\subsubsection{Pittsburgh Sleep Quality Index}

The occurrence of sleep disturbances was examined via the Pittsburgh Sleep Quality Index (PSQl; Buysse et al., 1989), an instrument previously validated in a comparable African population (Aloba et al., 2007). The PSQI results in seven subcomponents ranging from 0 to 3 with higher scores indicating more severe sleep disturbances. In a comparison of 80 western patients with primary insomnia compared to 45 healthy controls the instrument achieved a satisfactory internal consistency (Cronbach's Alpha $=0.85$ ) (Backhaus et al., 2002).

All participants were examined by a nurse at the day of cortisol measurements. Physiological parameters like the body mass index (BMI), body temperature, and pulse frequency were recorded. Exclusion criteria were current medication, current disease, psychiatric conditions (other than co-morbid depression symptoms and alcohol abuse), severe malnutrition and a generally bad health constitution (viz., a history of frequently displayed acute and chronic diseases accompanied by moderate malnutrition and a generally poor subjective well-being).

\subsection{Study protocol}

While the study was conducted, participants followed a strictly standardized routine (for a schematic depiction of the schedule see Fig. 1): After arrival early in the morning, directly from their home (travel time approximately 10$40 \mathrm{~min}$ ), participants were instructed on how to deliver saliva samples via Salivette sampling devices (Sarstedt, Numbrecht, Germany). The purpose of the investigation and all necessary procedures were explained in detail and written informed consent was obtained. To acquire a valid baseline measurement and to mirror expected cortisol fluctuations in the morning, saliva samples were collected at $07: 30 \mathrm{~h}$, 08:00 h, 08:30 h, and 09:30 h. For the rest of the day, saliva samples were obtained every $2 \mathrm{~h}$, at $11: 30 \mathrm{~h}, 13: 30 \mathrm{~h}$, $15: 30 \mathrm{~h}, 17: 30 \mathrm{~h}, 19: 30 \mathrm{~h}$, and $21: 00 \mathrm{~h}$. Standardized meals were ingested at $09: 35 \mathrm{~h}, 13: 35 \mathrm{~h}$, and $19: 35 \mathrm{~h}$, subsequent to the measurements. The $1.5 \mathrm{~h}$ interval between meals and the subsequent sample collection prevented potential effects of the food intake from affecting cortisol levels (Gibson et al., 1999). Smokers were permitted to smoke at $11: 35 \mathrm{~h}$, directly after lunch, and at $17: 35 \mathrm{~h}$, also subsequent to sample collection. Although smoking was standardized, smokers were excluded from the endocrinological analysis due to the influence of nicotine on cortisol release (Badrick 


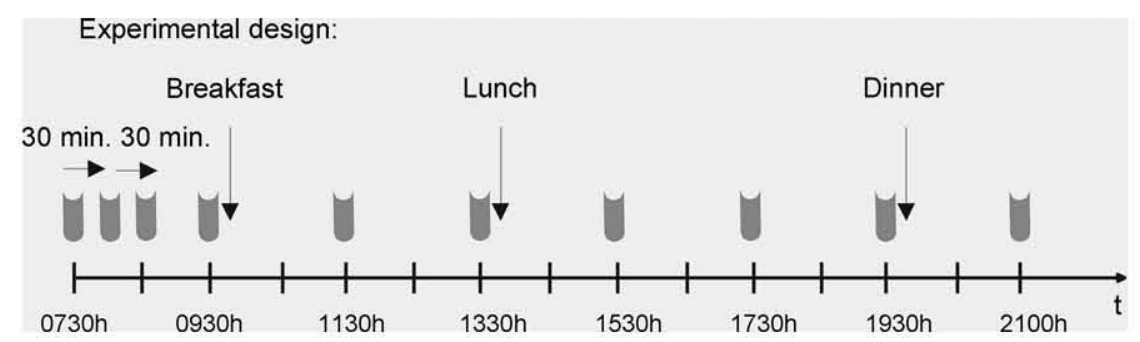

Figure 1 Experimental design of the study. Subjects arrived around 06:30 h. After the study procedure was explained in detail and written informed consent was obtained, measurements began with a circumstantial measurement of morning cortisol at 07:30 h, $08: 00 \mathrm{~h}, 08: 30 \mathrm{~h}$ and $09: 30 \mathrm{~h}$. Afterwards subject received breakfast and further saliva samples were obtained at $11: 30 \mathrm{~h}$ and at $13: 30 \mathrm{~h}$. Subjects then received lunch and the afternoon saliva probes were obtained at $15: 30 \mathrm{~h}, 17: 30 \mathrm{~h}$ and $19: 30 \mathrm{~h}$. At $19: 35 \mathrm{~h}$ subjects received dinner. A last measurement at $21: 00 \mathrm{~h}$ finalized the study procedure. Throughout the day subjects were at rest.

et al., 2007). This procedure also assured that there were no data from tobacco-deprived smokers included in the present analyses. Each deviation from the study protocol was recorded.

\subsection{Subjects}

Out of 60 participants, 9 were excluded from further analysis for the following reasons: current medication $(n=1)$, current disease ( $n=3$ ), severe malnutrition indicated by a body mass index below $16(n=2)$, a generally bad health constitution $(n=1)$, a cortisol value more than three standard deviations above the mean in one (out of the 10) measurements $(n=1)$, and cortisol levels under the detection limit on every measurement $(n=1)$. To ensure a comparison between subjects suffering from current PTSD and healthy traumatized controls, eight additional subjects who fulfilled the diagnostic criteria of lifetime PTSD but not of current PTSD were excluded from the analyses. Of the remaining 43 participants, 24 fulfilled the criteria of current PTSD according to DSM-IV (American Psychiatric Association, 1994). The remaining 19 participants never fulfilled the diagnostic criteria of PTSD and constituted the control group.

The participants' mean age was 38.0 years (S.D. $=7.84$, range $22-55$ years) in the PTSD group and 35.7 years (S.D. $=3.23$, range $31-41$ years) in controls. Both groups were comparable in age, educational status, marital status, and religion. Traumatization of participants largely took place a decade ago: $64.1 \%$ of participants stated that they experienced their "worst traumatic event" before or during 1994 (more precisely during the Rwandan genocide in 1994), $89.7 \%$ before 1997 . Subjects were between 9 and 43 years old when they experienced their "worst traumatic event" (mean age 24.85 , S.D. $=7.60$ ).

Six participants regularly used alcohol (four in the control group, two in the PTSD group). None of the subjects consumed any other drugs. PTSD subjects seemed to be of worse health constitution than controls, with a tendency towards lower weight and higher heart rates. As would be expected, PTSD subjects had experienced more traumatic event types than controls, PTSD subjects $M=15.75$, controls $M=9.42$, $t(41)=-3.98, p=.0005$, and showed higher PTSD as well as anxiety and depression scores as indexed by the PDS and the HSCL- 25 subscales (see Table 1 for parameters indicating the health status as well as mean questionnaire values, standard deviations and internal consistency coefficients of clinical instruments for each group). Nine participants (three in the control group, six in the PTSD group) fulfilled additional criteria for a Major Depressive Disorder according to DSMIV. Seventeen participants showed either low $(n=13)$, medium $(n=3)$ or high ( $n=1)$ suicidality, with higher suicidality in participants suffering from PTSD, $\chi_{3}^{2}(N=42)=9.23, p=.03$. PTSD subjects reported worse sleep than controls, as measured by the sum score of the PSQI, $t(41)=-2.54$, $p=.02$. Analyzing PSQI subscales revealed that participants with PTSD showed distinctive sleep disorders such as poor sleep quality, $t(41)=-2.17, p=.04$, sleep disturbances, $t(41)=-2.05, p=.05$, and daytime dysfunction, $t(41)=$ $-2.45, p=.02$. Mean sleep latency (time to fall asleep) was $41 \mathrm{~min}$ in the control group (S.D. $=38 \mathrm{~min}$ ) and $83 \mathrm{~min}$ (S.D. $=72 \mathrm{~min}$ ) in the PTSD group with a tendency towards longer sleep latencies in PTSD patients, $t(34.36)=-1.89$, $p=.07$. Thirteen participants were smokers and therefore excluded from the endocrinological analysis. Of the remaining subjects that were incorporated in the endocrinological analysis, 17 belonged to the PTSD group and 13 to the control group.

\subsection{Analysis of saliva samples}

Following each measurement, saliva samples were immediately stored at $-18{ }^{\circ} \mathrm{C}$. The day after completion of the study protocol, all samples were thawed and the saliva was spun down with manually operated centrifuges (Hettich, Tuttlingen, Germany). Afterwards, the spun saliva extract was stored at $+4{ }^{\circ} \mathrm{C}$ for 3 days and then brought to the laboratory of the ETH Zurich for analysis. This procedure was carried out to guarantee the stability of cortisol (Groschl et al., 2001; Garde and Hansen, 2005).

Saliva cortisol levels were measured using a competitive bead-based assay. Undiluted saliva or cortisol standard dilutions were incubated overnight in 96-well round-bottom plates with appropriate amounts of cortisol-BSA-conjugated polystyrene beads and fluorescein isothiocyanate (FITC)-conjugated rabbit anti-cortisol antibody (HTB192, Chromaprobe, Maryland Heights, MO, USA) at room temperature. After incubation, beads were washed and resuspended in phosphate-buffered saline, and analyzed on a flow cytometer (LSR II, BD Immunocytometry Systems, San Jose, CA, USA). The median fluorescence intensity is inversely proportional 
Table 1 Population characteristics for each group.

\begin{tabular}{lllll} 
Control group $(n=19)$ & & PTSD group $(n=24)$ & $\begin{array}{l}\text {-Value } \\
\text { Cronbach's } \\
\text { Alpha }\end{array}$ \\
\hline
\end{tabular}

Health status

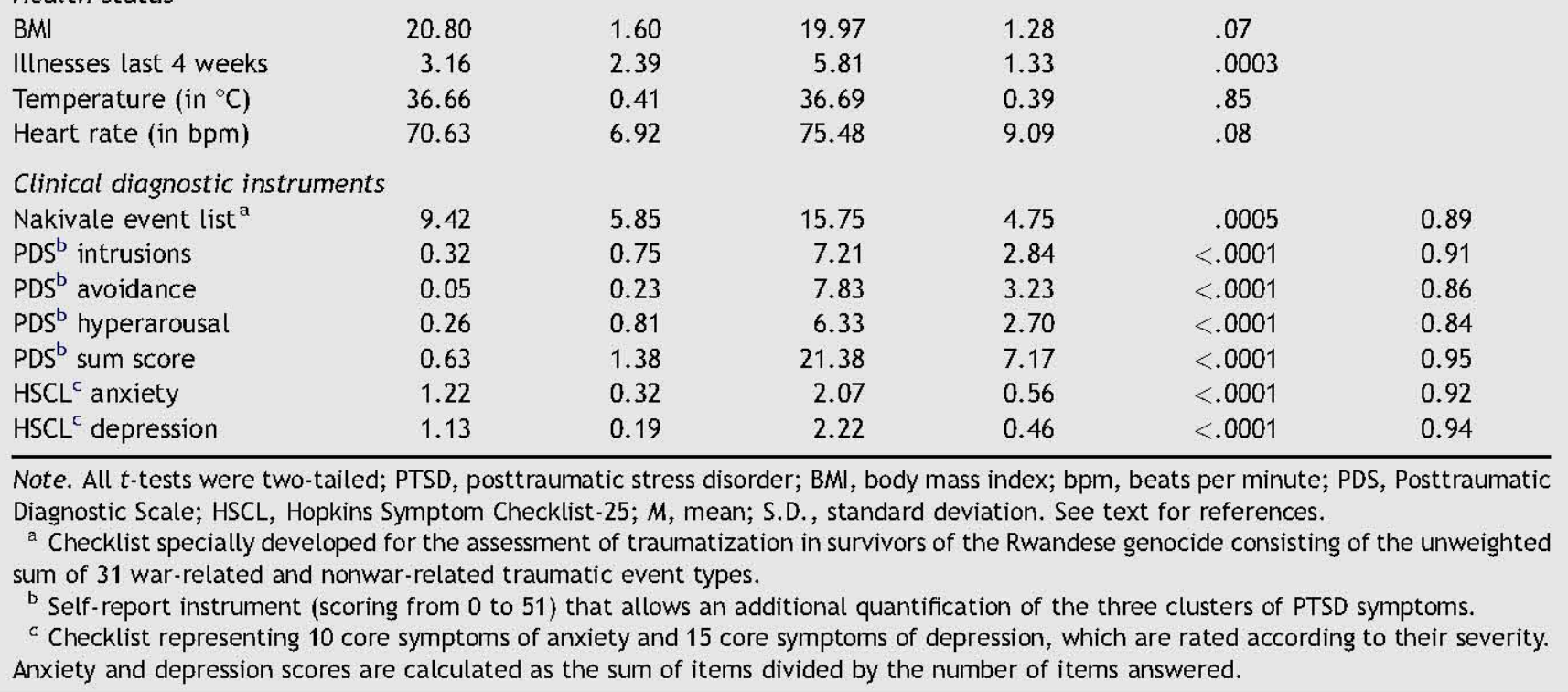

to the amount of cortisol in the sample. Intra- and inter-assay variance were $5.4 \%$ and $10.7 \%$, respectively. Antibody crossreactivity with other relevant steroids was $4.0 \%$ (testosterone) and $0.9 \%$ (progesterone), respectively.

\subsection{Statistical analysis}

Group differences in population characteristics and clinical parameters as well as in sleep disturbances were compared using $t$-tests. An investigation of a putative dosage effect of multiple traumatic events on the probability of PTSD diagnosis was performed using a logistic regression model and the effect of the number of different traumatic events on PTSD symptom severity using a bivariate regression model. To reveal the possible relationship between cortisol data and other variables, correlations were calculated between cortisol values and age, BMI, time of awakening, extent of traumatization, year of subjectively rated worst traumatic experience, age at worst traumatic experience, sum score of the PDS, PDS subscales, depression and anxiety scores and the severity of sleeping disorders.

Cortisol dynamics were assessed using a mixed-effects model analysis of covariance, including a random intercept for each participant. Time of day was included as a linear covariate in order to enable statements about hypothesized drops in cortisol over the day and to take the difference in intervals between successive cortisol measurements into account. In addition, analyses were conducted with time of day as a factor (without any assumptions about the correlation of e.g. successive time points), as it is more common in the cortisol literature. The normality assumption was not fulfilled for the residuals of raw cortisol data. Therefore, in order to reveal significant differences in the diurnal profiles of cortisol release, permutation tests were conducted. Permutation tests on the residuals of submodels
(Freedman and Lane, 1983; Anderson and Legendre, 1998) are non-parametric statistical significance tests in which the distribution of the statistic of interest (here, $F$-values for each factor and interaction) under the null hypothesis is found by randomly permuting residuals from submodels a large number of times. For example, when investigating the significance of a Group $\times$ Time point interaction, the vectors of residuals under the partial model Group + Time were permuted randomly and afterwards added to the unpermuted fitted values before recording the $F$-value of the interaction Group $\times$ Time point using these sum data. This $F$-value would be calculated a large number of times, yielding its null distribution. If there were no relation between Group and cortisol at the specific time points, then the same values would have occurred irrespective of the diagnosis of the subject. The significance of the statistic can therefore be assessed by comparing the distribution of the $F$-values under randomly permuted residuals with the $F$-value stemming from the actual data. In each case, 1000 random permutations were conducted, and the original $F$-value was inserted in the empirical distribution of $F$-values arising from the permuted ANOVAs under the assumption of the null hypothesis that there are no systematic group differences. $p$-Values reported below are the difference between 1 and this percentile, such that an original $F$-value falling at the 95 th percentile in the resampled $F$-value distribution is considered significant at the .05 level and is reported as $p=.05$. Degrees of freedom are irrelevant in permutation tests and are not reported below.

Excluding subjects fulfilling DSM-IV criteria of Major Depressive Disorder or excluding alcohol users did not affect the results. We furthermore included covariates that were associated with cortisol in this data or previous research such as age, BMI, time of awakening, extent of traumatization, symptom severity of PTSD, depression and anxiety scores, 
and sleeping disorders. Since BMI was the only covariate that showed a trend towards a significant influence $(F=.22$, $p=.06$ ), this variable remained in the analyses, while all other covariates were not included in the final analysis.

All analyses were conducted using the statistical program $\mathrm{R}$ (version 2.6.1; R Development Core Team, 2007) with the packages nime (version 3.1-86; Pinheiro et al., 2008), lars (version 0.9-7; Hastie and Efron, 2007) and sna (version 1.5; Butts, 2007).

\section{Results}

\subsection{Cortisol data}

Raw cortisol data are summarized in Table 2. Using permutation tests, no main effects of Time as a continuous covariate, $F=13.92, p=.22$, or Group, $F=1.09, p=.58$, and no Group $\times$ Time interaction, $F=2.21, p=.13$, were detected (see Fig. 2). Also no correlations between cortisol data and age, BMI, time of awakening, cumulative exposure to traumatic events, year of subjectively rated worst traumatic experience, age at worst traumatic experience, sum score of the PDS, PDS subscales, depression and anxiety scores or sleeping disorders were observed. When Time was entered in the model as a factor, the main effect Time was significant, $F=7.21, p<.0001$, while the interaction Group $\times$ Time was again insignificant, $F=1.02, p=.43$. No influence of number of traumatic events experienced on mean daytime cortisol levels or on cortisol levels at the different time points was found, i.e., no building-block effect on cortisol was observed.

\subsection{Cumulative effect of number of traumatic event types on PTSD diagnosis and PTSD symptom severity}

A positive relationship between the number of different types of traumatic experiences and incidence of PTSD was found by comparing a model including the number of event types with an intercept-only model using a $\chi^{2}$-test, $\log (P(\mathrm{PTSD}) / P(1-\mathrm{PTSD}))=-2.58+0.22 *$ Nakivale event list, $\chi_{1}^{2}=13.18, p=.003$. Furthermore, a linear regression analysis discovered a significant relationship between traumatic load and current symptom severity of PTSD, PDS $=0.31+0.92 *$ Nakivale event list, $R_{a d j}^{2}=0.20$, ANOVA $p=.001$. No correlation however was found between PTSD severity and the year of subjectively rated worst traumatic experience or age at worst traumatic experience.

\section{Discussion}

Despite controlling for many factors previously proposed to influence cortisol secretion and despite standardizing the study protocol as much as possible, we found no differences in the diurnal profile of cortisol release between participants with PTSD and traumatized controls. While this study replicated the previously reported building-block effect (Neuner et al., 2004; Kolassa and Elbert, 2007) of the number of different traumatic event types experienced on the likelihood to develop PTSD, we did not detect a similar effect of cumulative traumata on cortisol profiles.

\subsection{Diurnal slope of cortisol secretion}

Even under maximally standardized conditions, we did not observe general PTSD-related differences in the cortisol profiles of our study population. Multiple methodological variables concerning study design and population argued to distort cortisol findings were already mentioned (Rasmusson et al., 2003). As our investigation followed a strictly standardized schedule and as potential confounding variables (influences of time of awakening, year of subjectively rated worst traumatic experience, age at worst traumatic experience, alcohol or drug consumption, depression and sleeping disorders) were statistically ruled out, it is unlikely that methodological factors might have overshadowed PTSDrelated alterations in cortisol release reported by other groups (Yehuda et al., 1996; Lauc et al., 2004; Rohleder

Table 2 Summary of means and standard deviations of raw salivary cortisol data (in $\mathrm{nmol} / \mathrm{l})^{\mathrm{a}}$.

\begin{tabular}{|c|c|c|c|c|c|c|}
\hline \multirow[t]{2}{*}{ Cortisol at } & \multicolumn{2}{|c|}{ Control group } & \multicolumn{2}{|c|}{ PTSD group } & \multicolumn{2}{|c|}{ Entire population } \\
\hline & $M$ & S.D. & $M$ & S.D. & $M$ & S.D. \\
\hline $07: 30 \mathrm{~h}$ & 20.53 & 10.57 & 24.00 & 9.44 & 22.53 & 9.88 \\
\hline $08: 00 \mathrm{~h}$ & 15.89 & 8.08 & 15.96 & 9.77 & 15.93 & 8.92 \\
\hline $08: 30 \mathrm{~h}$ & 9.80 & 3.58 & 15.78 & 10.44 & 13.19 & 8.63 \\
\hline $09: 30 \mathrm{~h}$ & 9.42 & 6.89 & 10.50 & 6.63 & 10.03 & 6.65 \\
\hline $11: 30 \mathrm{~h}$ & 12.08 & 7.01 & 15.64 & 7.96 & 14.10 & 7.65 \\
\hline $13: 30 \mathrm{~h}$ & 12.10 & 8.09 & 15.42 & 8.72 & 13.93 & 8.47 \\
\hline $15: 30 \mathrm{~h}$ & 10.64 & 4.59 & 14.26 & 6.93 & 12.64 & 6.17 \\
\hline $17: 30 \mathrm{~h}$ & 10.37 & 5.79 & 11.58 & 8.84 & 11.06 & 7.58 \\
\hline $19: 30 \mathrm{~h}$ & 11.35 & 6.23 & 11.03 & 5.29 & 11.17 & 5.61 \\
\hline $21: 00 \mathrm{~h}$ & 12.63 & 8.93 & 10.42 & 7.06 & 11.38 & 7.86 \\
\hline
\end{tabular}

Note. PTSD, posttraumatic stress disorder; $M$, mean; S.D., standard deviation.

${ }^{a}$ For cautious comparison. The IBL Hamburg quotes following normal ranges for saliva cortisol in 110 healthy adult western subjects- 0 $1.5 \mathrm{~h}$ after awakening: $5.1-40.2 \mathrm{nmol} / \mathrm{l}(M d n=18.9), 1.5-3 \mathrm{~h}$ after awakening: $3.6-28.4 \mathrm{nmol} / \mathrm{l}(M d n=11.8), 3-6 \mathrm{~h}$ after awakening: 2.1-15.7 nmol/l $(M d n=6.7), 6-9 \mathrm{~h}$ after awakening: $1.8-12.1 \mathrm{nmol} / /(M d n=5.5), 9-15 \mathrm{~h}$ after awakening: $0.9-9.2 \mathrm{nmol} / \mathrm{l}(M d n=3.3)$. 


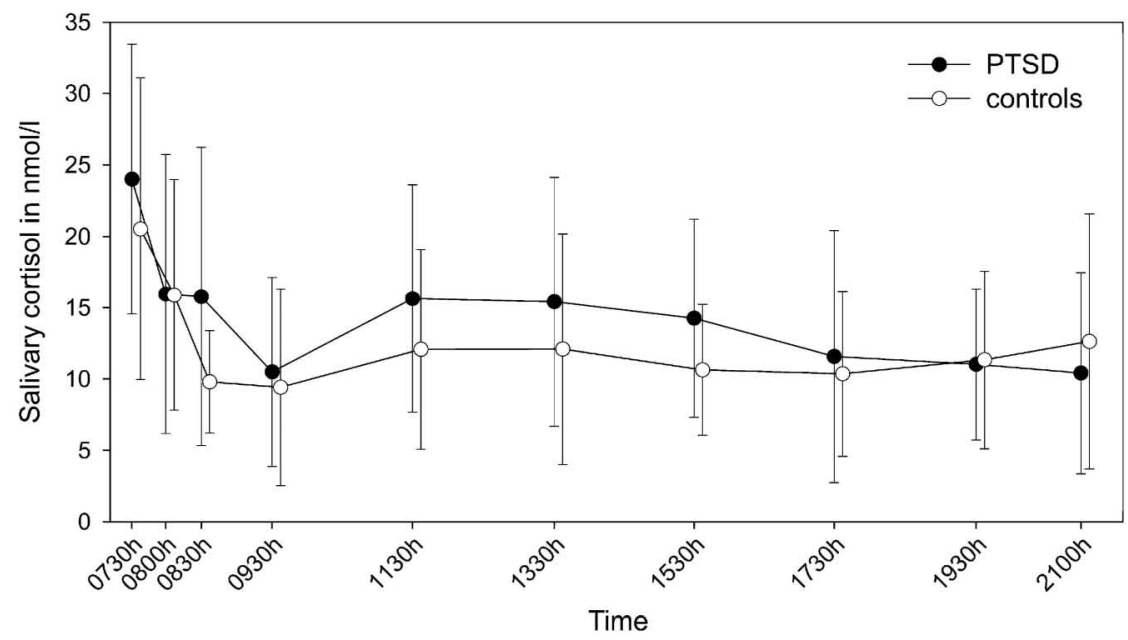

Figure 2 Comparison of the diurnal pattern of salivary cortisol (in nmol/l) release between the PTSD group and traumatized controls. Depicted are means and standard deviations. As BMI was the only covariate that showed a trend towards a significant influence $(F=.22$, $p=.06$ ), this variable was included in the analysis. There was neither a main effect of Time, $F=13.92, p=.22$, nor of Group, $F=1.09$, $p=.58$ and no interaction of Group $\times$ Time, $F=2.21, p=.13$. Note. PTSD, posttraumatic stress disorder; BMI, body mass index.

et al., 2004; Inslicht et al., 2006). In healthy populations a substantial inter-individual variation in cortisol profiles that might even exceed the intra-individual variability was already reported (Wüst et al., 2000; Ranjit et al., 2005). In light of this substantial variance and the absence of PTSDrelated alterations in cortisol secretion in our study and previous work (Young and Breslau, 2004; Young et al., 2004), some unknown factors influencing cortisol secretion apparently still remain to be identified.

Concerning our results, one approach to explain the differences between results and primary hypotheses might rely on the disadvantaged living conditions of the study participants. At the time of investigation all of them were living under poor sanitary and hygienic conditions. Participants engaged in physically strenuous fieldwork and may not have been sufficiently nourished. In previous research, reports of lowered cortisol levels in PTSD mainly have been based on subjects living under relatively stable conditions during the time of investigation (Yehuda et al., 1996, 2005; Lauc et al., 2004; Rohleder et al., 2004). Elevated cortisol values, on the other hand, were reported in PTSD patients currently living under trauma-related stress (Inslicht et al., 2006). In healthy subjects there are several reports that a disadvantaged milieu might alter the cortisol secretion (Steptoe et al., 2003; Cohen et al., 2006). Our results in conjunction with previous reports (Young and Breslau, 2004; Young et al., 2004) indicate that stressful living conditions might affect PTSDrelated alterations in cortisol release as well. However, this assumption so far remains mainly speculative, as our study included no external control group and no actual assessment of current life stress. Therefore, an influence of disadvantaged living conditions on PTSD-related alterations in the cortisol release should be systematically considered in future research.

Another factor that might generally influence PTSDrelated alterations in cortisol secretion might be the gender of participants. Studies reporting decreased cortisol levels in PTSD predominantly investigated male subjects (Yehuda et al., 1996; Lauc et al., 2004) or geriatric mixed gender populations with presumably postmenopausal women (Yehuda et al., 2005). Conversely, opposing results - no differences at all (Altemus et al., 2003; Young and Breslau, 2004; Young et al., 2004) and elevated cortisol levels (Inslicht et al. , 2006) - have been reported in females. As our investigation was confined to a pure male population, we are not able to make any assertions about potential gender differences in the cortisol secretion in this specific population.

Finally, our strict exclusion criteria led to a relatively small sample size. Although the sample size is comparable to the ones used previously (Meewisse et al., 2007), future studies may need to employ more participants, especially in the light of the large inter-individual variation in cortisol discussed above.

\subsection{Building-block effects}

The previous finding of a building-block effect of traumatization on the probability of PTSD diagnosis as well as on the severity of PTSD symptoms (Neuner et al., 2004) was replicated. There was a strong correlation between the number of different traumatic events reported by the subject and the diagnosis as well as symptom severity of PTSD. The finding that the accumulation of different kinds of traumata seems particularly to enhance the probability of developing PTSD has also been reported in other settings (Dohrenwend et al., 2006; Neuner et al., 2006). With respect to endocrinological data, no correlation was found between cortisol values and the extent of traumatization, i.e., no evidence for a buildingblock effect of trauma load on cortisol levels could be found.

\section{Conclusions and future perspectives}

In spite of maximal standardization, we did not find any influence of traumatization on diurnal cortisol secretion. Future studies should pay particular attention to possible confounding factors such as current life stress, address a possible gender effect - which has not yet been systematically 
investigated - and ensure a sufficiently large sample size to take large inter-individual variance into account.

\section{Role of the funding source}

Funding for this study was provided by the German Research Foundation (DFG); the DFG had no further role in study design, in the collection, analysis and interpretation of data, in the writing of the report and in the decision to submit the paper for publication.

\section{Conflict of interest statement}

All authors declare that they have no conflicts of interest including any financial, personal or other relationship with other people or organizations that could have inappropriately influenced their work.

\section{Acknowledgements}

We would like to thank Michael Bauer and Patience Onyut, who supported the data collection on-site and Christian Stoppel, who kindly assisted with the preparation and proof-reading of the manuscript.

\section{References}

Aloba, O.O., Adewuya, A.O., Ola, B.A., Mapayi, B.M., 2007. Validity of the Pittsburgh Sleep Quality Index (PSQI) among Nigerian university students. Sleep. Med. 8, 266-270.

Altemus, M., Cloitre, M., Dhabhar, F.S., 2003. Enhanced cellular immune response in women with PTSD related to childhood abuse. Am. J. Psychiatry 160, 1705-1707.

American Psychiatric Association, 1994. Diagnostic and statistical manual of mental disorders, 4th edition. American Psychiatric Press, Washington, DC.

Anderson, M.J., Legendre, P., 1998. An empirical comparison of permutation methods for tests of partial regression coefficients in a linear model. J. Statist. Comput. Simul. 62, 271-303.

Backhaus, J., Junghanns, K., Broocks, A., Riemann, D., Hohagen, F., 2002. Test-retest reliability and validity of the Pittsburgh Sleep Quality Index in primary insomnia. J. Psychosom. Res. 53 (3), 737-740.

Badrick, E., Kirschbaum, C., Kumari, M., 2007. The relationship between smoking status and cortisol secretion. J. Clin. Endocrinol. Metab. 92, 819-824.

Butts, C.T., 2007. Sna: tools for social network analysis. R package version 1.5. http://erzuli.ss.uci.edu/R.stuff.

Buysse, D.J., Reynolds 3rd, C.F., Monk, T.H., Berman, S.R., Kupfer, D.J., 1989. The Pittsburgh Sleep Quality Index: a new instrument for psychiatric practice and research. Psychiatry Res. 28, 193-213.

Cohen, S., Schwartz, J.E., Epel, E., Kirschbaum, C., Sidney, S., Seeman, T., 2006. Socioeconomic status, race and diumal cortisol decline in the coronary artery risk development in young adults (CARDIA) study. Psychosom. Med. 68, 41-50.

Derogatis, L.R., Lipman, R.S., Rickels, K., Uhlenhuth, E.H., Covi, L., 1974. The Hopkins Symptom Checklist (HSCL): a self-report symptom inventory. Behav. Sci. 19, 1-15.

Dohrenwend, B.P., Turner, J.B., Turse, N.A., Adams, B.G., Koenen, K.C., Marshall, R., 2006. The psychological risks of Vietnam for U.S. verterans: a revisit with new data and methods. Science 313 , 979-982.
Ertl, V., 2005. Reliability and validity of the assessment of posttraumatic stress disorder in an East-African refugee-camp. Master Thesis. Full text available under http://www.ub.uni-konstanz. de/kops/volltexte/2007/4062/.

Foa, E.B., Cashman, L., Jaycox, L., Perry, K., 1997. The validation of a self-report measure of posttraumatic stress disorder: the Posttraumatic Diagnostic Scale. Psychol. Assess. 9, 445-451.

Freedman, D., Lane, D., 1983. A nonstochastic interpretation of reported significance levels. J. Bus. Econ. Statist. 1, 292-298.

Friedman, M.J., Jalowiec, J., McHugo, G., Wang, S., McDonagh, A., 2007. Adult sexual abuse is associated with elevated neurohormone levels among women with PTSD due to childhood sexual abuse. J. Trauma. Stress 20, 611-617.

Garde, A.H., Hansen, A.M., 2005. Long-term stability of salivary cortisol. Scand. J. Clin. Lab. Invest. 65, 433-436.

Gibson, E.L., Checkley, S., Papadopoulos, A., Poon, L., Daley, S., Wardle, J., 1999. Increased salivary cortisol reliably induced by a protein-rich midday meal. Psychosom. Med. 61, 214-224.

Groschl, M., Wagner, R., Rauh, M., Dorr, H.G., 2001. Stability of salivary steroids: the influences of storage, food and dental care. Steroids $66,737-741$.

Hastie, T., Efron, B., 2007. Lars: least angle regression, lasso and forward stagewise. $R$ package version 0.9-7. http://www-stat. stanford.edu/ hastie/Papers/\#LARS.

Inslicht, S.S., Marmar, C.R., Neylan, T.C., Metzler, T.J., Hart, S.L., Otte, C., McCaslin, S.E., Larkin, G.L., Hyman, K.B., Baum, A., 2006. Increased cortisol in women with intimate partner violence-related posttraumatic stress disorder. Psychoneuroendocrinology $31,825-838$.

Kaspers, F.A., Scholz, O.B., 2004. Stress-induced increase in morning cortisol variance. Stress Health 20, 127-129.

Kirschbaum, C., Hellhammer, D.H., 1994. Salivary cortisol in psychoneuroendocrine research: recent developments and applications. Psychoneuroendocrinology 19 (4), 313-333.

Kirschbaum, C., Kudielka, B.M., Gaab, J., Schommer, N.C., Hellhammer, D.H., 1999. Impact of gender, menstrual cycle phase, and oral contraceptives on the activity of the hypothalamus-pituitaryadrenal axis. Psychosom. Med. 61 (2), 154-162.

Kirschbaum, C., Prüssner, J.C., Stone, A.A., Federenko, I., Gaab, J., Lintz, D., Schommer, N., Hellhammer, D.H., 1995. Persistent high cortisol responses to repeated psychological stress in a subpopulation of healthy men. Psychosom. Med. 57 (5), 468-474.

Kolassa, I.T., Elbert, T.R., 2007. Structural and functional neuroplasticity in relation to traumatic stress. Curr. Dir. Psychol. Sci. 16, 321-325.

Lauc, G., Zvonar, K., Vuksic-Mihaljevic, Z., Flögel, M., 2004. Short communication: post-awakening changes in salivary cortisol in veterans with and without PTSD. Stress Health 20, 99-102.

Lee, B., Kaaya, S.F., Mbwambo, J.K., Smith-Fawzi, M.C., Leshabari, M.T., 2008. Detecting depressive disorder with the Hopkins Symptom Checklist-25 in Tanzania. Int. J. Soc. Psychiatry 54 (1), 7-20.

Luecken, L.J., Suarez, E.C., Kuhn, C.M., Barefoot, J.C., Blumenthal, J.A., Siegler, I.C., Williams, R.B., 1997. Stress in employed women: impact of marital status and children at home on neurohormone output and home strain. Psychosom. Med. 59, 352359.

McEwen, B.S., 2000. Allostasis and allostatic load. Implications for neuropsychopharmacology. Neuropsychopharmacology 22 (2), 108-124.

McEwen, B.S., 2005. Stressed or stressed out. What is the difference? J. Psychiatry Neurosci. 30 (5), 315-318.

Meewisse, M.L., Reitsma, J.B., de Vries, G.J., Gersons, B.P., Olff, M., 2007. Cortisol and post-traumatic stress disorder in adults: systematic review and meta-analysis. Br. J. Psychiatry 191, 387392.

Neuner, F., Onyut, P.L., Ertl, V., Odenwald, M., Schauer, E., Elbert, T., 2008. Treatment of posttraumatic stress disorder through trained lay therapists counselors in an African refugee settlement in 
Uganda-a randomized controlled trial. J. Clin. Consult. Psychol. $76(4), 686-694$.

Neuner, F., Schauer, E., Catani, C., Ruf, M., Elbert, T., 2006. Posttsunami stress: a study of posttraumatic stress disorder in children living in three severely affected regions in Sri Lanka. J. Trauma. Stress 19, 339-347.

Neuner, F., Schauer, M., Karunakara, U., Klaschik, C., Robert, C., Elbert, T., 2004. Psychological trauma and evidence for enhanced vulnerability for posttraumatic stress disorder through previous trauma among West Nile refugees. BMC Psychiatry 4, 34.

Odenwald, M., Lingenfelder, B., Schauer, M., Neuner, F., Rockstroh, B., Hinkel, H., Elbert, T., 2007. Screening for post traumatic stress disorder among Somali ex-combatants: a validation study. Confl. Health 1, 10.

Onyut, P.L., Neuner, F., Schauer, E., Ertl, V., Odenwald, M., Schauer, M., Elbert, T., 2004. The Nakivale Camp Mental Health Project: Building local competency for psychological assistance to traumatized refugees. Intervention 2 (2), 90-107.

Pinheiro, J., Bates, D., DebRoy, S., Sarkar, D., R Core team, 2008. Nlme: linear and nonlinear mixed effects models. $R$ package version 3.1-86.

Powell, L.H., Lovallo, W.R., Matthews, K.A., Meyer, P., Midgley, A.R., Baum, A., Stone, A.A., Underwood, L., McCann, J.J., Janikula Herro, K., Ory, M.G., 2002. Physiologic markers of chronic stress in premenopausal, middle-aged women. Psychosom. Med. 64, 502-509.

R Development Core Team, 2007. R: a language and environment for statistical computing. Vienna, Austria.

Ranjit, N., Young, E.A., Raghunathan, T.E., Kaplan, G.A., 2005. Modeling cortisol rhythms in a population-based study. Psychoneuroendocrinology 30, 615-624.

Rasmusson, A.M., Vythilingam, M., Morgan 3rd, C.A., 2003. The neuroendocrinology of posttraumatic stress disorder: new directions. CNS Spectr. 8 651-656, 665-657.

Rohleder, N., Joksimovic, L., Wolf, J.M., Kirschbaum, C., 2004. Hypocortisolism and increased glucocorticoid sensitivity of pro- inflammatory cytokine production in Bosnian war refugees with posttraumatic stress disorder. Biol. Psychiatry 55, 745-751.

Roy, M.P., Kirschbaum, C., Steptoe, A., 2001. Psychological, cardiovascular, and metabolic correlates of individual differences in cortisol stress recovery in young men. Psychoneuroendocrinology 26, 375-391.

Sheehan, D.V., Lecrubier, Y., Sheehan, K.H., Amorim, P., Janavs, J., Weiller, E., Hergueta, T., Baker, R., Dunbar, G.C., 1998. The MiniInternational Neuropsychiatric Interview (M.I.N.I.): the development and validation of a structured diagnostic psychiatric interview for DSM-IV and ICD-10. J. Clin. Psychiatry 59 (Suppl. 20), 2233 quiz 34-57.

Smith, S.M., Vale, W.W., 2006. The role of the hypothalamic-pituitary-adrenal axis in neuroendocrine responses to stress. Dialogues Clin. Neurosci. 8, 383-395.

Steptoe, A., Kunz-Erbrecht, S., Owen, N., Feldman, P. J., Willemsen, G., Kirschbaum, C., Marmot, M., 2003. Socioeconomic status and stress-related biological responses over the working day. Psychosom. Med. 65, 461-470.

Wüst, S., Wolf, J., Hellhammer, D.H., Federenko, I., Schommer, N., Kirschbaum, C., 2000. The cortisol awakening response-normal values and confounds. Noise Health 2 (7), 79-88.

Yehuda, R., Golier, J.A., Kaufman, S., 2005. Circadian rhythm of salivary cortisol in Holocaust survivors with and without PTSD. Am. J. Psychiatry 162, 998-1000.

Yehuda, R., Teicher, M.H., Trestman, R.L., Levengood, R.A., Siever, L.J., 1996. Cortisol regulation in posttraumatic stress disorder and major depression: a chronobiological analysis. Biol. Psychiatry 40, 79-88.

Young, E.A., Breslau, N., 2004. Saliva cortisol in posttraumatic stress disorder: a community epidemiologic study. Biol. Psychiatry 56, 205-209.

Young, E.A., Tolman, R., Witkowski, K., Kaplan, G., 2004. Salivary cortisol and posttraumatic stress disorder in a low-income community sample of women. Biol. Psychiatry 55, 621-626. 